\title{
Antropologia Médica: Elementos Conceituais e Metodológicos para uma Abordagem da Saúde e da Doença
}

\author{
Medical Anthropology: Conceptual and Methodological Elements for an \\ Approach to Health and Disease
}

\author{
Elizabeth Uchôa ${ }^{1,2}$ \\ Jean Michel Vidal ${ }^{2}$
}

\author{
UCHÔA, E. E VIDAL, F. M. Medical Anthropology: Conceptual and Methodological Elements \\ for an Approach to Health and Disease. Cad. Saúde Públ., Rio de Faneiro, 10 (4): 497-504, \\ Oct/Dec, 1994. \\ This paper discusses the relevance, specificity, and potential of the anthropological approach to \\ health and illness. Medical anthropology is shown as complementary to other approaches that \\ currently deal with public health problems. The impact of social and cultural factors on health- \\ related perceptions and behaviors is illustrated and commented. A conceptual and \\ methodological framework is also proposed to systematize the study of representations and \\ practices of communities in the area of public health. The specific contribution of the \\ anthropological approach is discussed in terms of the effectiveness of public health programs.
}

Key words: Medical Anthropology; Perceptions and Behaviors; Public Health

\section{ABORDAGEM ANTROPOLÓGICA. PERTINÊNCIA, ESPECIFICIDADES E POTENCIALIDADES}

Os trabalhos antropológicos na área de saúde tem aumentado progressivamente, existindo hoje vasta literatura sobre o assunto. Não pretendemos analisar neste artigo as contribuições específicas dos diferentes autores ou examinar as várias abordagens que delimitam o campo da Antropologia Médica. O trabalho tem objetivos mais limitados. Ele visa a demonstrar a pertinência do discurso antropológico na abordagem da saúde e da doença.

Sabemos hoje que noções, como as de saúde e doença, aparentemente simples referem-se, de fato, a fenômenos complexos que conjugam fatores biológicos, sociológicos, econômicos, ambientais e culturais. A complexidade do ob-

1 Centro de Pesquisas René Rachou, Fundação Oswaldo Cruz. Av. Augusto de Lima, 1715, Belo Horizonte, MG, 30190-002, Brasil.

2 Unité de Recherche Psychosociale, Centre de Recherche de l'Hôpital Douglas. 6875 Bd. La Salle, Montréal, Canada. jeto, assim definido, transparece na multiplicação de discursos sobre a saúde que coexistem atualmente, cada um privilegiando diferentes fatores e sugerindo estratégias de intervenção e de pesquisa também diversas.

$\mathrm{O}$ discurso antropológico aponta os limites e a insuficiência da tecnologia biomédica quando se trata de mudar de forma permanente o estado de saúde de uma população. Ele nos revela que o estado de saúde de uma população é associado ao seu modo de vida e ao seu universo social e cultural. A antropologia médica se inscreve, assim, numa relação de complementaridade com a epidemiologia e com a sociologia da saúde.

A epidemiologia estuda a distribuição das doenças (ou de condições relacionadas à saúde) em populações e busca os determinantes dessa distribuição. Nos estudos epidemiológicos predominam as abordagens sobre os comportamentos dos indivíduos, e métodos quantitativos são utilizados. A prevalência ou a incidência de uma certa patologia e as características de indivíduos apresentando ou não essa patologia são determinadas, com o objetivo de identificar os perfis de distribuição da pato- 
logia e grupos ou fatores de risco. $\mathrm{Na}$ abordagem sociológica, os problemas de saúde são apreendidos em sua dimensão social e não individual. A sociologia da saúde investiga a determinação que exercem os contextos social e institucional sobre as enfermidades e os comportamentos delas decorrentes. A antropologia considera que a saúde e o que se relaciona a ela (conhecimento do risco, idéias sobre prevenção, noções sobre causalidade, ideias sobre tratamentos apropriados, etc.) são fenômenos culturalmente construídos e culturalmente interpretados (Nichter, 1989). A perspectiva qualitativa é empregada para identificar e analisar a mediação que exercem os fatores sociais e culturais na construção de formas características de pensar e agir frente à saude e à doença. Integrando uma apreensão da dimensão cultural, a antropologia médica vem, ao lado da sociologia da saúde e da epidemiologia, contribuir para ampliar o contexto que deve ser levado em consideração na leitura dos processos patológicos.

Uma profunda dicotomia entre métodos quantitativos e qualitativos ainda afeta o conjunto das ciências sociais; essa dicotomia obscurece a complementaridade dessas duas estratégias de pesquisa, cada uma tendo seus pontos fortes e suas fraquezas (Létourneau, 1989). Diversos autores (Minayo \& Sanches, 1993; Létourneau, 1989) sugerem que as perspectivas quantitativas e qualitativas deveriam ser encaradas como perspectivas complementares, como fases sequenciais de um mesmo processo. As estratégias qualitativas indicam o que é importante estudar em um dado contexto sóciocultural, permitem identificar variáveis pertinentes e formular hipóteses culturalmente apropriadas. As pesquisas quantitativas são construidas a partir de amostras representativas do grupo estudado e permitem testar essas hipóteses. $\mathrm{O}$ reconhecimento dos pontos fortes e dos limites de cada uma dessas perspectivas suporta o movimento atual, que advoga a adoção de abordagens multi e transdisciplinares em pesquisas no campo da saúde (Rosenfield, 1992). Nestes últimos anos, vários autores escreveram sobre o interesse, as dificuldades decorrentes e as consequências metodológicas de uma conjugação entre as perspectivas quantitativa e qualitativa (Hundt \& Forman, 1993). O ponto comum desses trabalhos é a constatação do enriquecimento gerado pela conjugação das duas perspectivas.

Neste artigo, a antropologia médica é apresentada como uma perspectiva complementar e enriquecedora para a abordagem dos problemas de saúde pública. Começamos por examinar a influência do contexto social e cultural sobre "as maneiras de pensar e de agir" das populações frente aos seus problemas de saúde e, a partir daí, situamos a contribuição específica da abordagem antropológica. Apresentamos, em seguida, alguns elementos conceituais e metodológicos que intervêm de maneira fundamental na construção do conhecimento antropológico em saúde e propomos um quadro de referência para o estudo sistemático das representações e comportamentos associados à saúde e à doença.

\section{DOENCA E CULTURA: MANEIRAS DE PENSAR E MANEIRAS DE AGIR}

As informações culturais têm sido, na maioria das vezes, consideradas irrelevantes para as intervenções preventivas e terapêuticas na área da saúde (Good \& DelVecchio Good, 1980). Em geral, são tidas como essenciais unicamente aquelas referentes ao diagnóstico biomédico. Todos os outros dados, em particular aqueles referentes ao impacto dos fatores sociais e culturais, são avaliados como acessórias (Kleinman, 1987).

Contrariando esse ponto de vista, estudos recentes demonstram a grande influência que exercem os universos social e cultural sobre a adoção de comportamentos de prevenção ou de risco e sobre a utilização dos serviços de saúde (Taylor et al., 1987). O grande investimento que tem sido feito em campanhas de vacinação em diferentes países do mundo (WHA, 1977, 1982) e o relativo sucesso dessas campanhas em alguns países africanos (Unger, 1991) pode ser citado como um primeiro exemplo. Razões técnicas (cadeia fria não respeitada, pessoal paramédico pouco ou não formado, etc.) são geralmente evocadas para explicar as dificuldades que essas campanhas enfrentam. Devem também ser levados em consideração os fatores culturais que podem comprometer o sucesso dessas campanhas. Responsáveis técnicos e médicos formados pelos métodos científicos 
ocidentais ignoram muitas vezes a significação que as campanhas de vacinação podem ter para as populações visadas. Por exemplo, como elas percebem o fato de dar medicamentos a uma criança, aparentente sadia, que, em muitos casos, terá febre nas 24 horas seguintes à vacinação e, portanto, parecerá doente? Não é fácil convencer os pais da criança de que isso a protegerá de certas doenças no futuro. Tais campanhas não podem ser facilmente transportadas de um contexto a outro. Elas exigem que se levem em conta as particularidades culturais e os diferentes processos lógicos predominantes em cada contexto.

De modo geral, os programas de saúde partem do pressuposto de que a informação gera uma transformação automática dos comportamentos das populações frente às doenças. Essa abordagem negligencia os diferentes fatores sociais e culturais que intervêm na adoção desses comportamentos (Fincham, 1992).

Diversos autores ressaltam a grande influência que exercem a semiologia popular e as concepçôes culturais de causalidade sobre os comportamentos adotados frente às doenças (Nyamwaya, 1987; Green, 1992; Hielscher \& Sommerfield, 1985; Corin et al., 1992a). Segundo Green (1992), a causa principal das doenças sexualmente transmissíveis é, em várias sociedades africanas, percebida como a violação de normas que governam os comportamentos sexuais. Partindo dessa concepção, a população prefere recorrer ao tratamento com terapeutas tradicionais do que aos serviços médicos. Um outro bom exemplo dessa influência é fornecido pelo estudo de Agyepong (1992), que investigou as percepções e práticas frente à malária em uma comunidade de Gana. Esse autor mostrou: (1) que a palavra malária não era popularmente conhecida e que uma categoria popular "asra" englobava um complexo de sinais e sintomas muito semelhantes aos da malária, incluindo febre; (2) que muitos membros da comunidade não conectavam o vetor à doença; a população acreditava que o "asra" era causado por contato prolongado com o calor excessivo. Essa concepção etiológica eliminava quase toda possibilidade de prevenção, pois o sol está sempre presente e não há como evitálo. Essa doença era também considerada pela população incurável pela medicina, pois, com o tratamento, a doença desaparecia, mas voltava a reaparecer algum tempo mais tarde. $\mathrm{O}$ desconhecimento das causas de reinfecção levava à desvalorização dos efeitos do medicamento. Como conseqüência, a maioria das pessoas era tratada em casa, a cloroquina era pouco utilizada e geralmente em doses subterapêuticas.

Hielscher \& Sommerfield (1985) discutem a relação entre as concepções culturais das doenças e a utilização de recursos médicos em uma comunidade rural do Mali. Os autores descrevem a identificação da causa cultural como etapa fundamental do processo terapêutico. As concepções etiológicas populares dão significado aos diferentes episódios patológicos e determinam em grande medida as estratégias para lidar com eles. Hielscher \& Sommerfield (1985) ressaltam que não existe um conceito popular equivalente ao conceito de esquistossomose. Os diversos sinais e sintomas são percebidos pelas populações como entidades separadas e independentes. O estudo de Nyamwaya (1987) junto aos Pokot, do Kenia, demonstra igualmente a influência das concepções culturais de causalidade sobre a utilização das formas tradicionais e ocidentais de terapia. No entanto, a influência de outros fatores no processo decisório é também demonstrada pelo autor. Segundo Nyamwaya (1987), os Pokot seriam bastante pragmáticos e revelariam grande capacidade de integrar novas idéias e novas práticas; a eficácia comprovada de uma ou outra terapêutica seria fundamental tanto como princípio classificatório como na escolha do tratamento adequado.

Todos esses estudos revelam que os comportamentos de uma população frente a seus problemas de saúde, incluindo a utilização dos serviços médicos disponíveis, são construídos a partir de universos sócio-culturais específicos. Eles apontam a necessidade de enraizarem-se os programas de educação e o planejamento em saúde em conhecimento prévio das formas características de pensar e agir predominantes nas populações junto às quais se quer intervir (Hielscher \& Sommerfield, 1985; Nyamwaya, 1987; Nichter, 1989; Corin et al., 1989; Fincham, 1992; Agyepong, 1992; Green, 1992, Inecom, 1993). 


\section{MODELO MÉDICO - MODELOS CULTURAIS. ELEMENTOS CONCEITUAIS E METODOLÓGICOS PARA UMA ABORDAGEM ANTROPOLÓGICA DA SAÚDE E DA DOENÇA}

Segundo Scheper-Hugues \& Lock (1987), o dualismo cartesiano entre corpo e espírito seria o precursor imediato das concepções biomédicas contemporâneas de organismo humano e do pensamento materialista radical que caracteriza a biomedicina. Descartes teria legado às ciências naturais e sociais uma concepção mecanicista do corpo e de suas funções que sustenta uma visão reducionista dos fenômenos saúde e doença. A doença é ora vista como um problema físico ou mental, ora como biológico ou psicossocial, mas raramente como fenômeno multidimensional. A fragmentação do objeto gera a fragmentação das abordagens. A descontinuidade entre as diferentes abordagens retarda a apreensão multidimensional do objeto.

A contribuição da antropologia é aqui extremamente importante. Ela ressitua nossas premissas básicas no horizonte epistemológico ocidental, tornando possível uma perspectiva crítica frente a nossas "verdades" mais fundamentais e favorecendo a construção de um novo paradigma para a abordagem da saúde e da doença.

Com o desenvolvimento da corrente interpretativa em antropologia, surge uma nova concepção da relação entre indivíduo e cultura e tornase possível uma verdadeira integração da dimensão contextual na abordagem dos problemas de saúde. C. Geertz, que se situa na origem dessa corrente, concebe a cultura como o universo de símbolos e significados que permite aos indivíduos de um grupo interpretar a experiência e guiar sua ações (Geertz, 1973: pp). Segundo ele, a cultura fornece modelos "de" e modelos "para" a construção das realidades sociais e psicológicas. Para Geertz, a cultura é o contexto no qual os diferentes eventos se tornam inteligíveis. Essa concepção estabelece ligação entre as formas de pensar e as formas de agir dos indivíduos de um grupo, ou seja, entre os aspectos cognitivos e pragmáticos da vida humana e ressalta a importância da cultura na construção de todo fenômeno humano. Nessa perspectiva considera-se que as percepções, as interpretações e as ações, até mesmo no campo da saúde, são culturalmente construídas.

Os trabalhos desenvolvidos pelo Grupo de Harvard e, em particular, pelos professores Arthur Kleinman e Byron Good, que se situam entre os principais representantes da corrente interpretativa em antropologia médica, fornecem os elementos-chave de um quadro teórico e metodológico para análise dos fatores culturais que intervêm no campo da saúde. Esses trabalhos ressaltam a importância de considerar que as desordens, sejam elas orgânicas ou psicológicas, só nos são acessíveis por meio da mediação cultural; "a desordem é sempre interpretada pelo doente, pelo médico e pelas famílias" (Kleinman \& Good, 1985).

A distinção paradigmática estabelecida por Eisenberg (1977) entre a "doença processo" (disease) e a "doença experiência" (illness) é o elemento-chave desse grupo de estudos. A "doença processo" (disease) refere-se às anormalidades de estrutura ou funcionamento de orgãos ou sistemas, e a "doença experiêcia" (illness), à experiência subjetiva do mal-estar sentido pelo doente. Nessa perspectiva, a experiência da doença não é vista como simples reflexo do processo patológico no sentido biomédico do termo. Considera-se que ela conjuga normas, valores e expectativas, tanto individuais como coletivas, e se expressa em formas específicas de pensar e agir.

Kleinman (1980), inspirando-se em Geertz (1973), afirma que a cultura fornece modelos "de" e "para" os comportamentos humanos relativos à saúde e à doença. Segundo Kleinman, todas as atividades de cuidados em saúde são respostas socialmente organizadas frente às doenças e podem ser estudadas como um sistema cultural: health care system. Todo "sistema de cuidados em saúde" seria constituído pela interação de três setores diferentes (profissional, tradicional e popular). Cada setor veiculando crenças e normas de conduta específicas e legitimando diferentes alternativas terapêuticas.

Kleinman (1980) elaborou o conceito de "modelo explicativo" (explanatory model) para estudar os traços cognitivos e os problemas de comunicação associados às atividades de saúde. Segundo esse autor, o modelo explicativo é constituído por noções elaboradas a partir de 
episódios de doenças e em referência aos tratamentos que foram utilizados. Kleinman (1980) distingue "os modelos explicativos" dos profissionais e os "modelos explicativos" utilizados pelos doentes e suas famílias. Esses modelos se enraízam em diferentes setores do sistema médico e veiculam crenças, normas de conduta e expectativas específicas. A grande contribuição desses instrumentos analíticos é que eles permitem abordar sistematicamente, e em seus aspectos plural e dinâmico, o conjunto de valores, crenças e normas de conduta predominantes no campo da saúde. $\mathrm{O}$ estudo de modelos explicativos empregados por diferentes categorias de pessoas (profissionais, doentes, famílias e outros) permite uma avaliação da distância que separa os modelos médicos e não médicos, o exame da interação entre eles e a análise dos problemas de comunicação que surgem do encontro entre modelos culturais e modelos médicos durante as atividades clínica, educativa ou de pesquisa. $\mathrm{O}$ conhecimento dos modelos explicativos, que predominam em um grupo, facilita a comunicação com os indivíduos desse grupo e permite a realização de intervenções que sejam compreensíveis e aceitáveis para eles, duas condições essenciais para o sucesso de qualquer programa de saúde.

O modelo de análise de redes semânticas (semantic network analysis), desenvolvido por Good (1977) e Good \& DelVecchio Good (1980, 1982), abre o caminho para a compreensão dos diferentes fatores que participam da construção de "realidades médicas". Esses autores enfatizam a diversidade de modelos que suportam, em uma sociedade, a construção cultural dos problemas de saúde e os esforços terapêuticos para resolvê-los. Eles ressaltam que toda prática terapêutica é eminentemente interpretativa e implica constante trabalho de tradução, de decodificação e de negociação entre diferentes sistemas semânticos. Segundo Good (1977) e Good \& Delvecchio Good (1980, 1982), a significação dos episódios patológicos seria construída em redes de significações (semantic network illness), por meio das quais elementos cognitivos, afetivos e experienciais se articulam sobre o universo das relações sociais e das configurações culturais. Essas redes de símbolos associadas a doenças particulares em dada sociedade seriam utilizadas pelos indiví- duos para interpretar o vivido, articular a experiência e exprimi-la de forma socialmente legítima. $\mathrm{O}$ interesse desse modelo aparece mais claramente quando se considera que não existe correspondência termo a termo entre os diagnósticos profissionais, que geralmente orientam os programas de saúde, e os diagnósticos populares, que orientam as representações e comportamentos das comunidades (Hielscher \& Sommerfield, 1985). A percepção do que é relevante e problemático, do que causa ou evita um problema, do tipo de ação que esse problema requer é, para os profissionais de saúde, determinada pelo corpo de conhecimentos biomédicos, mas, para os indivíduos de uma comunidade, é determinada pelas redes de símbolos que articulam conceitos biomédicos e culturais e determinam formas características de pensar e de agir frente a um problema de saúde específico.

Os trabalhos de A. Kleinman, B. Good e M. J. Delvecchio Good reinscrevem as crenças, as normas de comportamento e as expectativas referentes às doenças no contexto mais amplo de normas e valores que predominam em uma sociedade. Eles questionam o "naturalismo" das interpretações biomédicas, favorecem maior tolerância frente a outras formas de pensar e agir diante da doença e, em conseqüência, abrem novos caminhos para o reconhecimento e análise dos processos culturais que mediatizam a construção das representações e comportamentos em saúde.

O modelo de análise dos "sistemas de signos, significados e ações" elaborado por Corin et al. (1989, 1990, 1992a, 1992b, 1993) se inscreve, em linhas gerais, como um prolongamento dos trabalhos do Grupo de Harvard, já descritos. Todavia, ele permite maior sistematização dos diferentes elementos do contexto (dinâmica social, códigos culturais centrais, conceito de pessoa, etc.) que intervêm efetivamente na identificação do que é problemático, na decisão de tratar ou não um problema e na escolha do terapeuta apropriado.

Embora o modelo de análise dos "sistemas de signos, significados e ações" tenha sido inicialmente empregado na área da saúde mental, sua contribuição potencial para outras áreas da saúde, parece indiscutível. Muito pouco se conhece sobre sinais e sintomas considerados 
relevantes por populações específicas ou sobre interpretações culturalmente associadas a um determinado problema e menos ainda se conhece sobre os comportamentos característicos nos quais se traduzem essas percepções e interpretações. O modelo de análise dos "sistemas de signos, significados e ações" visa precisamente ao conhecimento sistemático das maneiras de pensar e de agir de populações junto às quais se quer intervir; ele constitui um instrumento privilegiado para a investigação antropológica das representações e comportamentos predominantes no campo das grandes endemias.

Esse modelo de análise é construído a partir de duas premissas básicas: (1) cada comunidade constrói de maneira específica o universo dos problemas de saúde, marcando principalmente tal ou tal sintoma, privilegiando tal ou tal explicação e encorajando certos tipos de reações e ações; (2) existe continuidade entre a maneira pela qual uma comunidade percebe e interpreta seus problemas de saúde e os procedimentos que ela desenvolve para resolvê-los; essa construção específica é ligada às características sócio-culturais da comunidade e às condições macroscópicas de contexto (Corin et al., 1990).

A proposta metodológica de Corin et al. (1990)é de inverter o procedimento geralmente utilizado nos estudos sobre representações e partir do nível pragmático para remontar ao nível semântico. Os comportamentos concretos de indivíduos servem de ponto de partida para um estudo que tenta identificar as lógicas conceituais subjacentes a esses comportamentos e os diferentes fatores que intervêm na concretização destas lógicas em situações particulares (Corin et al., 1989). A experiência anterior dos autores (Corin et al., 1992b) revela que existe grande distância entre os discursos que descrevem uma doença em termos gerais e a maneira pela qual são percebidas e interpretadas as ocorrências concretas dessa doença; daí a escolha de focalizar as ações concretas de indivíduos particulares, frente a problemas específicos, e, por essa via, remontar ao universo de percepções e representações.

A análise dos "sistemas de signos, significados e ações" é feita a partir do estudo das práticas dos atores apreendidas por histórias concretas. Uma fase preliminar do estudo visa à delimitação do campo semântico que cobre um determinado problema. São identificados termos locais e formuladas descrições significativas que servirão para identificar casos. Para cada caso identificado, diferentes questões tentam reconstruir de maneira detalhada os comportamentos e sintomas associados à doença, as interpretações feitas por diferentes categorias de pessoas, os tratamentos realizados e/ou que deveriam idealmente ser realizados. As entrevistas são gravadas, transcritas e codificadas em função de registros de conteúdo e de categorias analíticas. Toda informação é compilada em quadros que permitem a identificação das categorias de informação dominantes. Cada entrevista é, em seguida, indexada com a ajuda de um software (por exemplo, Sato ou Ethnograph) para permitir a rápida extração dos textos correspondentes às diferentes categorias de informação e a identificação do informante. Um primeiro nível de análise visa a identificar "os sistemas de signos, significados e ações", ou seja: 1) os diferentes tipos de signos associados à identificação de um determinado problema, à gravidade desse problema ou à necessidade de tratamento; 2) as explicações privilegiadas frente a esses signos e 3 ) as reações e ações que são desencadeadas por esses signos. Um segundo nível de análise procura examinar as articulações entre "os sistemas de signos, significados e ações" e determinar o impacto específico de diferentes elementos do contexto pessoal, social e cultural sobre a construção e a evolução das reações e dos comportamentos (Corin et al., 1989, 1990, 1992a, 1992b, 1993; Uchôa et al., 1993).

O modelo de análise "dos sistemas de signos, de significados e de ações" possibilita a sistematização da investigação antropológica na área das grandes endemias. Ele permite o conhecimento das lógicas conceituais que organizam o campo das representações culturais, associadas por populações específicas a cada endemia, e de elementos do contexto (experiência pessoal, exigências profissionais, hábitos culturais, fatores ambientais, etc.) que podem influenciar a tradução dessas representações em comportamentos concretos (de risco, de proteção, frente aos recursos de saúde existentes).

O emprego desse modelo em estudos de diferentes problemas de saúde pública contribui- 
rá, certamente, para o refinamento da pesquisa nessa área e para a reformulação das questões relativas ao planejamento e à organização de programas de saúde, no sentido de maior adequação das intervenções às características sociais e culturais das populações junto às quais se quer intervir.

\section{CONCLUSÃO}

$\mathrm{Na}$ perspectiva antropológica, o universo sócio-cultural do doente é visto não mais como obstáculo maior à efetividade dos programas e práticas terapêuticas, mas como o contexto onde se enraízam as concepções sobre as doenças, as explicações fornecidas e os comportamentos diante delas. Essa perspectiva reorienta a percepção dos aspectos relacionados à efetividade das intervenções em saúde. Se considerarmos que a efetividade de um programa de saúde depende da extensão em que a população aceita, utiliza e participa desse programa, essa efetividade parece, assim, ser dependente do conhecimento prévio das maneiras características de pensar e agir associadas à saúde nessa população e da habilidade do programa em integrar esse conhecimento (Inecom, 1993).

A antropologia desenvolveu importante aparelhagem conceitual e metodológica para o estudo sistemático das maneiras culturais de pensar e de agir associadas à saúde. Ela permite examinar as relações (interações e contradições) entre os modelos de prática, que suportam a organização dos serviços, os programas de prevenção e as intervenções terapêuticas, e os modelos culturais dos usuários. A partir daí, ela fornece parâmetros para a reformulação da questão da adequação sócio-cultural dos diferentes programas de saúde.

\section{RESUMO}

\section{UCHÔA, E. \& VIDAL J. M. Antropologia \\ Médica: Elementos Conceituais e \\ Metodológicos para uma Abordagem da}

Saúde e da Doença. Cad. Saúde Públ., Rio de Janeiro, 10 (4): 497-504, out/dez, 1994.

$\mathrm{O}$ artigo discute a pertinência, as especificidades e as potencialidades da perspectiva antropológica na abordagem da saúde e da doença. A antropologia médica é apresentada como uma perspectiva complementar e enriquecedora na abordagem dos problemas de saúde pública. A influência do universo social e cultural sobre as percepções e ações em saúde é exemplificada e comentada em referência a estudos realizados junto a diferentes populações. Elementos conceituais e metodológicos centrais são apresentados e integrados à proposta de sistematização do estudo "das maneiras de pensar e de agir" face a diferentes problemas de saúde. A contribuição específica da abordagem antropológica é discutida em termos da efetividade dos programas saúde pública.

Palavras-Chave: Antropologia Médica; Percepções e Comportamentos; Saúde Pública

\section{REFERÊNCIAS BIBLIOGRÁFICAS}

AGYEPONG, I. A., 1992. Malaria ethnomedical perceptions and practice in an Adangbe farming community and implications for control. Social Sciences and Medicine, 35: 131-137.

CORIN, E.; UCHÔA, E.; BIBEAU, G. \& HARNOIS, G., 1989. Les Attitudes Dans le Champ de la Santé Mentale. Repères Théoriques et Méthodologiques pour une Étude Ethnographique et Comparative. Rapport Technique. Montréal: Centre de Recherche de l'Hôpital Douglas, Centre Collaborateur OMS. (Mimeo.)

CORIN, E.; BIBEAU, G.; MARTIN, J. C. \& LAPLANTE, R., 1990. Comprendre pour Soigner Autrement. Repères pour Régionaliser les Services de Santé Mentale. Montréal: Presses de l'Université de Montréal.

CORIN, E.; UCHÔA, E.; BIBEAU, G.; KOUMARÉ, B.; COULIBALY, B.; COULIBALY, M.; MOUNKORO, P. \& SISSOKO, M., 1992a. La place de la culture dans la psychiatrie africaine d'au- 
jourd'hui. Paramètres pour un cadre de références. Psychopathologie Africaine, 24: 149-181.

CORIN, E.; UCHÔA, E.; BIBEAU, G. \& KOUMARE, B., 1992b. Articulation et variations des systèmes de signes, de sens et d'actions. Psychopathologie Africaine, 24: 183-204.

CORIN, E.; BIBEAU, G. \& UCHÔA, E., 1993. Eléments d'une sémiologie anthropologique des troubles psychiques chez les Bambara, Bwa et Soninké du Mali. Anthropologie et Sociétés, 17: 125-156.

EISENBERG, L., 1977. Disease and illness: distinctions between profesiional and popular ideas of sickness. Culture, Medicine and Psychiatry, 1: 09-23.

FINCHAM, S., 1992. Community health promotion programs. Social Sciences and Medicine, 35: 239-249.

GEERTZ, C., 1973. The Interpretation of Cultures. New York: Basic Books Inc. Publishers.

GOOD, B., 1977. The heart of what's the matter: the semantics of illness in Iran. Culture, Medicine and Psychiatry, 1: 25-58.

GOOD, B. \& DELVECCHIO GOOD, M. J., 1980. The meaning of symptoms: a cultural hermeneutic model for clinical practice. In: The Relevance of Social Science for Medicine (L. Eisenberg \& A. Kleinman, eds.), pp. 165-196, Dordrechet: Reideil Publishing Co.

, 1982. Toward a meaning-centered analysis as popular illness categories: "FrightIllness" and "Heart Distress" in Iran. In: Cultural Conceptions of Mental Health and Therapy (A. J. Marsella \& G. White, eds.), pp. 141-166, Dordrechet: D. Reidel Publishing Co.

GREEN, E. C., 1992. Sexuallly trasmited disease, ethnomedicine and health policy in Africa. Social Sciences and Medicine, 35: 121-130.

HIELSCHER, S. \& SOMMERFIELD, J., 1985. Concepts of illness and the utilization of health care services in a rural Malien Village. Social Sciences and Medicine, 21: 397-400.

HUNDT, G. A. \& FORMAN, M.,R., 1993. Interfacing anthropology and epidemiology: the bedouin arab infant feeding study. Social Sciences and Medicine, 36: 957-964.

INECOM, 1993. The International Network for Cultural Epidemiology and Community Mental Health. Montréal: WHO. (Collaborating Centre for Research and Training in Mental Health). (Mimeo.)

KLEINMAN, A., 1980. Patients and Healers in the Context of Cultures. An Exploration of Boderland between Anthropology and Psychiatry. Berkeley/Los Angeles: University of California Press.
, 1987. Anthropology and psychiatry. The role of culture in cross-cultural research on illness. British Fournal of Psychiatry, 151: 447454.

KLEINMAN, A. \& GOOD, B. (Eds.), 1985. Culture and Depression: Studies in Anthropology and Cross-Cultural Psychiatry of Affect and Disorder. Berkeley: University of California Press.

LÉTOURNEAU, G., 1989. Létude comparative du veillissement. In: Veillir à Travers du Monde ( $\mathrm{R}$. Santerre \& G. Létourneau, eds.), pp. 113-130, Québec: Presses Universitaires de Laval.

MINAYO, M. C. S. \& SANCHES, O., 1993. Quantitativo-qualitativo: oposição ou complementaridade? Cadernos de Saúde Púbica, 9: 239262.

NICHTER, M., 1989. Anthropology and International Health: South Asian Case Studies. Dordrechet: Kluwer Publications.

NYAMWAYA, D., 1987. A Case study of interaction between indigenous and western medicine among the Pokot of Kenya. Social Sciences and Medicine, 25: 1277-1287.

ROSENFIELD, P. L., 1992. The potential of transdisciplinary research for sustaining and extending linkages between health and social sciences. Social Sciences and Medicine, 35: 1343-1357.

SCHEPER-HUGUES, N. \& LOCK, M., 1987. The mindful body. Medical Anthropology Quaterly, 1: 06-42.

TAYLOR, P.; CHANDIWANA, S. K.; GOVERE, J. M. \& CHOMBO, F., 1987. Knowlwdge attitudes and practices in relation to schistosomiasis in a rural community. Social Sciences and Medicine, 24: 607-611.

UCHÔA, E.; CORIN, E.; KOUMARE, B. \& BIBEAU, G., 1993. Représentations culturelles et disqualification sociale: l'épilepsie dans trois groupes ethniques au Mali. Psychpathologie Africaine, 25: 33-57.

UNGER, J. P., 1991. Can intensive campaigns dynamize front line health services? The evaluation os an immunization campaign in thiès health district, Sénégal. Social Sciences and Medicine, 32: 249-259.

WHA (World Health Assembly), 1977. Resolution 30.53. World Health Assembly, may. (Mimeo.) , 1982. Resolution 35.51. World Health Assembly, may. (Mimeo.) 